\title{
Classical galactosemia in a Thai infant: case report and review of the literature
}

\author{
Thitima Ngoenmak ${ }^{\mathrm{a}}$, Julintorn Somran ${ }^{\mathrm{b}}$, Chutima Phuaksaman ${ }^{\mathrm{a}}$, Jaruwat Khunrat ${ }^{\mathrm{c}}$ \\ ${ }^{a}$ Department of Pediatrics, Faculty of Medicine, Naresuan University, Phitsanulok 65000, Thailand \\ ${ }^{b}$ Department of Pathology, Faculty of Medicine, Naresuan University, Phitsanulok 65000, Thailand \\ 'Department of Radiology, Faculty of Medicine, Naresuan University, Phitsanulok 65000, Thailand
}

\begin{abstract}
Background: Classic galactosemia is an inherited disorder of galactose metabolism that is caused by a deficiency of galactose-1-phosphate uridyl transferase (GALT). As in other Asians, the prevalence of galactosemia in Thai people is very low. An accumulation of its toxic metabolites leads to acute neonatal toxicity and long-term complications.

Objective: To present the fourth known published case of classical galactosemia in a Thai infant and review the English language literature.

Method: A 4-month-old boy who was born into a Thai family with no history of consanguinity developed persistent jaundice, hepatosplenomegaly, and lethargy, since introduction to breast-feeding.

Result: Urine gas chromatography-mass spectrometry demonstrated a high level of galactose, galactitol, and galactonate. Liver biopsy confirmed severe hepatocellular damage and fibrosis. Breast-feeding was immediately replaced by a lactose-free diet and soy milk. His clinical features and subsequent laboratory measurements improved. Developmental delays and defects on speech presented at the last followed up.

Conclusion: Long-term complications are diet-independent and inevitable. However early recognition and immediate withdraw of galactose from the diet can prevent serious morbidity and mortality.
\end{abstract}

Keywords: Acute neonatal toxicity, classic galactosemia, galactose-1-phosphate uridyl transferase, long-term complications, urine gas chromatography-mass spectrometry

Galactosemia is an autosomal recessive genetic condition in which the body has a defect in galactose metabolism, the Leloir pathway. Three key enzymes in this pathway consist of galactokinase (GALK), galactose-1-phosphate uridyl transferase (GALT), and galactose-6-phosphate epimerase (GALE). Deficiency in any of three enzymes in this pathway causes an accumulation of toxic metabolites that leads to acute deterioration of multiple organ systems and long-term complications. GALT deficiency causes classic galactosemia, which has greater incidence than other types [1-6]. Most classic galactosemia presents clinically during the neonatal period, after galactose ingestion. Acute neonatal toxicity results in jaundice, hepatosplenomegaly, hepatocellular insufficiency, food intolerance, hypoglycemia, renal tubular dysfunction, muscle hypotonia, sepsis, and cataract [4].

Correspondence to: Thitima Ngoenmak, Department of Pediatrics, Faculty of medicine, Naresuan University, Amphur Muang, Phitsanulok 65000, Thailand.

E-mail: Thitiman@nu.ac.th
The prevalence of classical galactosemia, a consequence of GALT deficiency, varies greatly with different ethnicity. The prevalence of this disease is higher in people of European origin than in Asians. The estimated prevalence of classical galactosemia is 1:23,500 to 44,000 in UK, 1:50,000 in USA, 1:400,000 in Taiwan, and 1:700,000 in Japan [2, 7]. In Thailand, the exact prevalence of classical galactosemia is unidentified. The established newborn screening program covers only congenital hypothyroidism and phenylketonuria [8], but does not include galactosemia. Since 1988, there were to our knowledge only three Thai patients reported as diagnosed with galactosemia [9-12]. In the English language literature, the second and third cases were available to review.

We report a fourth case of classical galactosemia in a Thai male infant. He developed jaundice, hepatosplenomegaly, and lethargy within a few days after breast-feeding, and these features persisted until he was 4 months old. Clinical and laboratory improvement confirmed a good response to lactosefree diet. 
This retrospective study was approved by the Ethics and Institutional Review Committee and Board of Naresuan University.

\section{Case report}

A 4-month-old, Thai male infant was normally delivered at full term (40 weeks of gestational age) as a first son to nonconsanguineous mother. His family has no history of known inherited disease. Because of meconium aspiration syndrome, he had a poor Apgar score and was intubated for a short period. He started breast-feeding on his third day of life. Two days later, he developed jaundice, hepatosplenomegaly, and lethargy. He had blood group A, Rh positive and his mother's blood group was $\mathrm{O}, \mathrm{Rh}$ positive. A direct Coombs test was weakly positive, while an indirect Coombs test was negative. Cholestatic jaundice progressed poorly after he received total parental nutrition (TPN). When TPN was discontinued, serum level of total bilirubin and direct bilirubin minimally reduced. Sputum culture showed a growth of Acinetobacter baumannii and Enterobacter cloacae. He received antibiotics for 14 days. After his infectious problem resolved, he was referred to Naresuan University Hospital (NUH) because of persistent jaundice, hepatosplenomegaly, and lethargy.

On admission at NUH, his body weight was $7.6 \mathrm{~kg}$ and crown-heel length was $63 \mathrm{~cm}$. He was afebrile, lethargic, hypotonia, and icteric. His liver was $10 \mathrm{~cm}$ long, and splenic palpation was $10 \mathrm{~cm}$ below the costal margin. There was no cataract. Glucose- 6-phosphate dehydrogenase (G-6-PD) deficiency was confirmed by our laboratory, but the patient was asymptomatic. Serum total and direct bilirubin were $24.57 \mathrm{mg} \%$ and $12.79 \mathrm{mg} \%$ respectively. Plasma aspartate transaminase (397 U/L, reference range 5-40 U/L), alanine transaminase (109 U/L, reference range 5-40 U/L), and alkaline phosphatase $(740 \mathrm{U} / \mathrm{L}$, reference range 35-110 U/L) were significantly elevated. Liver biopsy was performed and revealed severe hepatocyte loss with giant cell transformation, hepatocellular cholestasis, intralobular fibrosis, portal fibrosis and ductular proliferation (Figure 1).

Metabolic screen for inborn errors of metabolism, including alpha-keto acids, cystine/homocystine, ferric chloride, mucopolysaccharides, and reducing substances was negative. Increased excretion of galactose, galactitol, and galactonate were discovered in his urine via gas chromatography-mass spectrometry (GCMS) (Figure 2). His diet was immediately changed from breast feeding to nonlactose diet and soy milk. Five months later, his clinical features and subsequent clinical laboratory measurements improved. The level of galactitol, and galactonate excretion in urine had declined. The liver injury was resolving, but portal fibrosis still presented (Figure 3). Recent follow up at 4 years old showed development delays, and there is a slow progression of growth. He can sit alone and walk with one hand held. Defects on speech and language development were detected. He can make "dada-mama" sounds, nonspecific sounds, and imitate speech sounds.
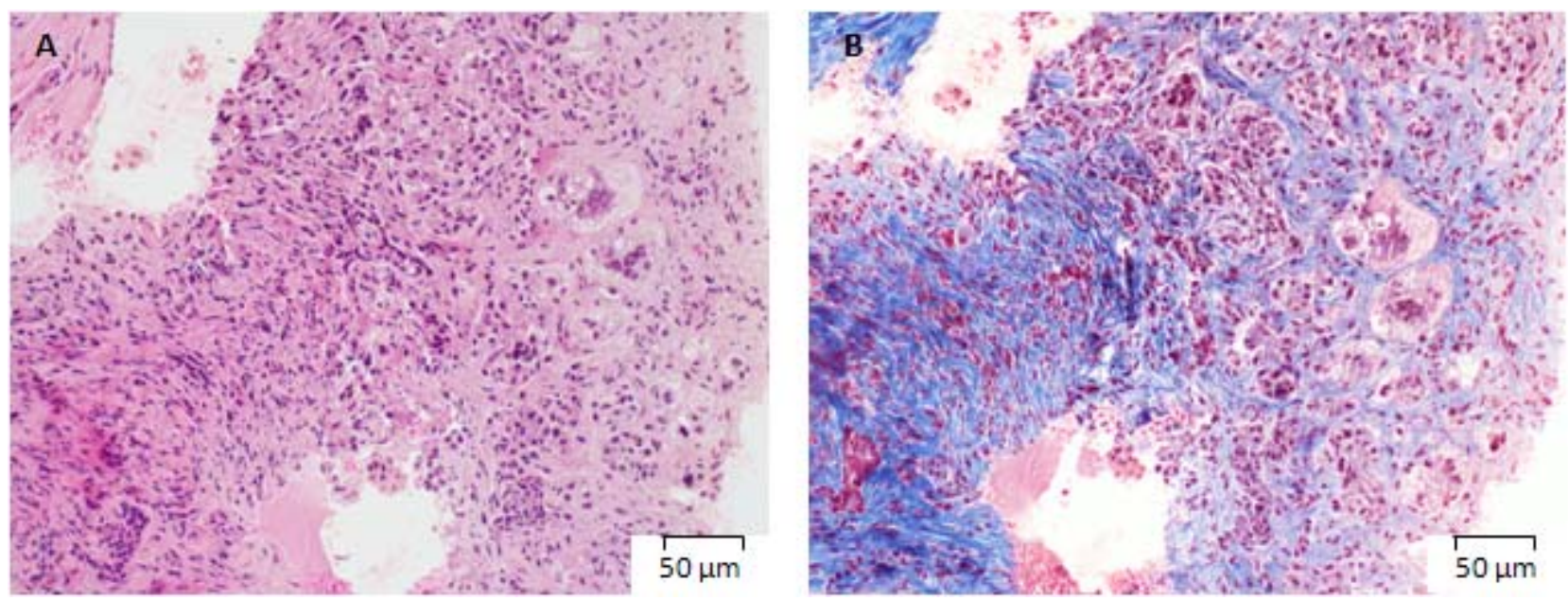

Figure 1. A. Hematoxylin and eosin staining $(\times 200)$. B. Masson trichrome staining $(\times 200)$. Severe hepatocellular damage with giant cell transformation, intralobular, and portal fibrosis 


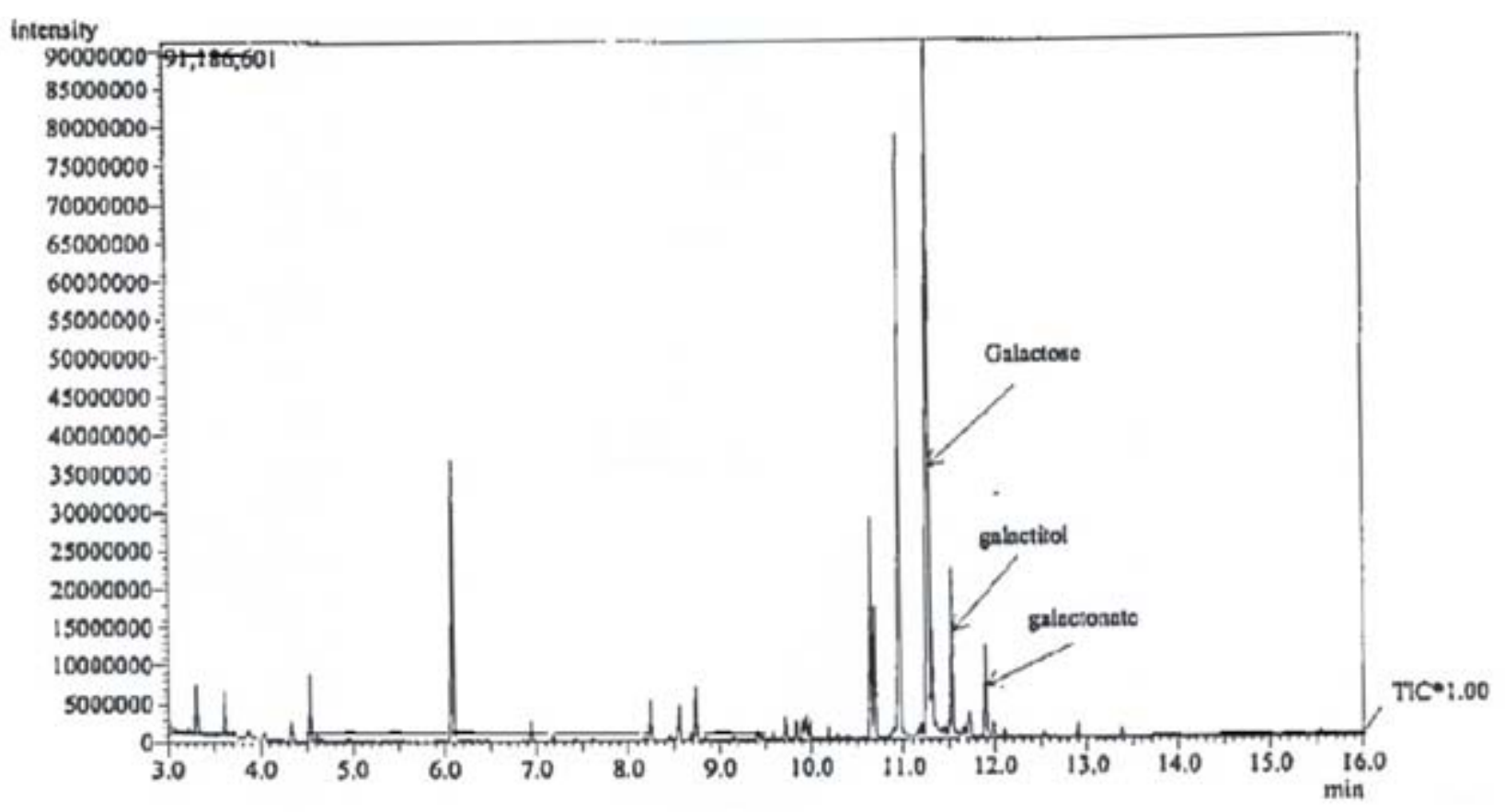

Figure 2. Gas chromatography-mass spectrometry demonstrates an increase excretion of galactose, galactitol, and galactonate in the patient's urine.
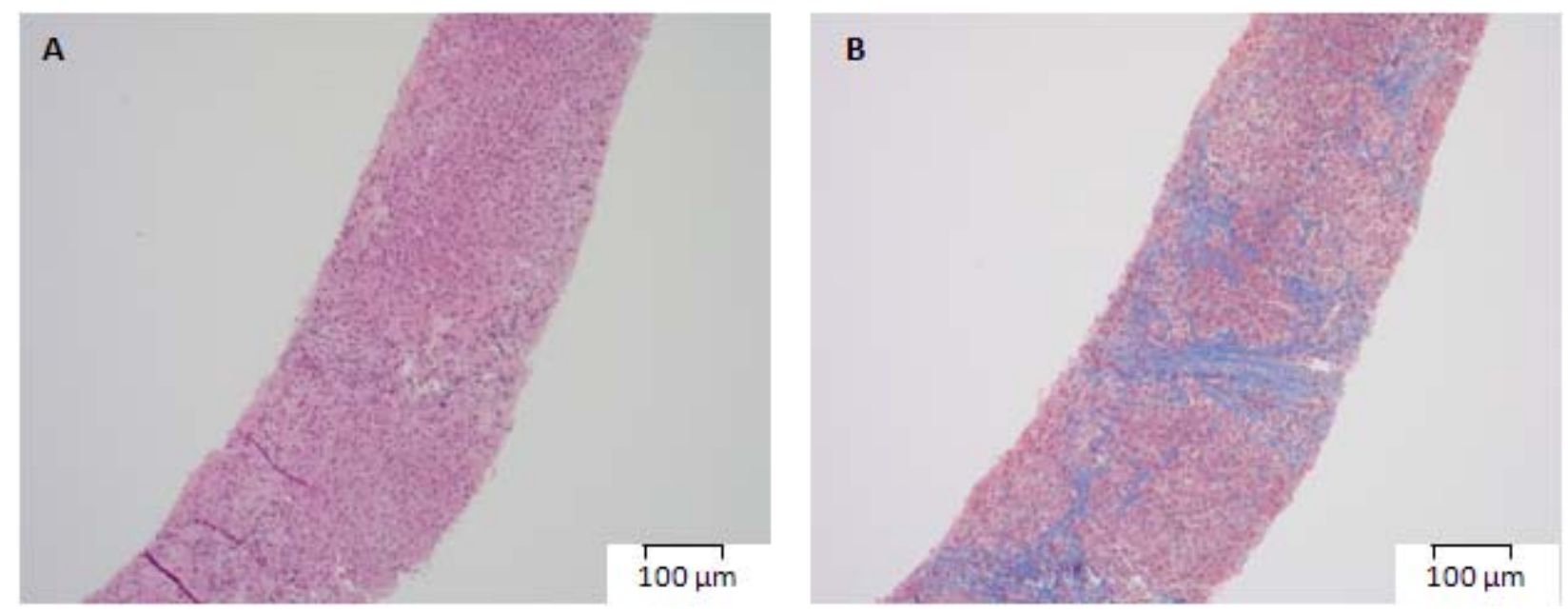

Figure 3. A. Hematoxylin and eosin staining $(\times 100)$ and B. Masson trichrome staining $(\times 100)$. Follow-up liver biopsy, after galactose restriction revealed no active liver damage, but residual fibrosis was demonstrated by Masson trichrome staining.

\section{Discussion}

We report a case of galactosemia in a 4-monthold Thai boy who is the fourth Thai patient reported in the English literature to our knowledge [9-11]. The clinical features and laboratory results of cases of galactosemia in Thais are compared as shown in Table 1. Our patient was born into a family without a history of consanguinity and/or known inherited disease. He developed jaundice on his fifth day of life or two days after introduction to breast-feeding. Many possible causes of neonatal jaundice were investigated. G6PD deficiency was a suspected cause of his jaundice, and sepsis and TPN induced liver injury. Although infection subsided and TPN was removed, his clinical features such as jaundice, hepatosplenomegaly, and lethargy persisted. The 
GCMS demonstrated an increased excretion of galactose, galactitol, and galactonate in his urine (Figure 2) that indicates an enzymatic defect in galactose metabolism. The measurement of galactose-1-phosphate uridyltransferase activity in erythrocytes, being the criterion standard diagnostic tool [4] for classic galactosemia was unavailable, and we had no genotype study results. The diagnosis of classic galactosemia in the present case was based on clinical features and accessible laboratory results.

Elevated blood level of galactose (gal) might be secondary or primary hypergalactosemia. Secondary hypergalactosemia presents in many abnormalities, including congenital hepatitis, congenital hepatic arteriovenous malformations, patent ductus venosus, tyrosinemia, type 1, citrin deficiency (citrullinemia, type 2 ), other metabolic-disorders producing hepatocellular disease and Fanconi-Bickel syndrome [3]. Based on the Leloir and alternative pathway as in Figure 4, primary hypergalactosemia disorder results from an enzymatic defect of galactose metabolism and is divided into three types, including GALK deficiency, GALT deficiency, and GALE deficiency [1-6]. The free galactose in plasma is subsequently changed into galactitol and galactonate through the alternative pathway of galactose metabolism. Subsequently galactose, galactitol, and galactonate excreted into urine that can be detected by GCMS. Accumulation of galactitol and galactonate destroy selected tissue leading to acute life-threatening disease and long-term complications. Acute neonatal toxicity effects multiple organs, including liver, kidney, and brain [2]. Long-term effects include chronic brain injury, cataract, and ovarian failure [3, 6].

Table 1. The clinical features and laboratory results of Thai galactosemia cases in English literature

\begin{tabular}{|c|c|c|c|}
\hline & Second case [11] & Third case [12] & Present case \\
\hline Age & 2 months & 11 days & 4 months \\
\hline Sex & Female & Male & Male \\
\hline Family History & Nonconsanguinity & Nonconsanguinity & Nonconsanguinity \\
\hline \multicolumn{4}{|l|}{ Clinical features } \\
\hline Poor feeding & Yes & Yes & Yes \\
\hline Vomiting & No & Yes & Yes \\
\hline Hepatomegaly & Yes & Yes & Yes \\
\hline Gram negative sepsis & No & No & Yes \\
\hline Lethargy and hypotonia & No & Yes & Yes \\
\hline Cataract & Yes & No & No \\
\hline \multicolumn{4}{|l|}{ Laboratory } \\
\hline Cholestasis jaundice & Yes & Yes & Yes \\
\hline Urine GC/MS & $\begin{array}{l}\text { Rising galactose, } \\
\text { galactitol, and } \\
\text { galactonate }\end{array}$ & $\begin{array}{l}\text { Rising galactose, } \\
\text { galactitol, and } \\
\text { galactonate }\end{array}$ & $\begin{array}{l}\text { Rising galactose, } \\
\text { galactitol, and } \\
\text { galactonate }\end{array}$ \\
\hline Genetic analysis & No & $\begin{array}{l}\text { Mutation of p.R259W } \\
\text { in exon } 8 \text { and p. E340K } \\
\text { in exon } 10\end{array}$ & No \\
\hline Liver biopsy finding & No & No & $\begin{array}{l}\text { Severe hepatocyte loss } \\
\text { with giant cell } \\
\text { transformation and } \\
\text { cholestasis }\end{array}$ \\
\hline Long-term complications & Unavailable & Unavailable & $\begin{array}{l}\text { Delayed development } \\
\text { and defects on speech }\end{array}$ \\
\hline
\end{tabular}




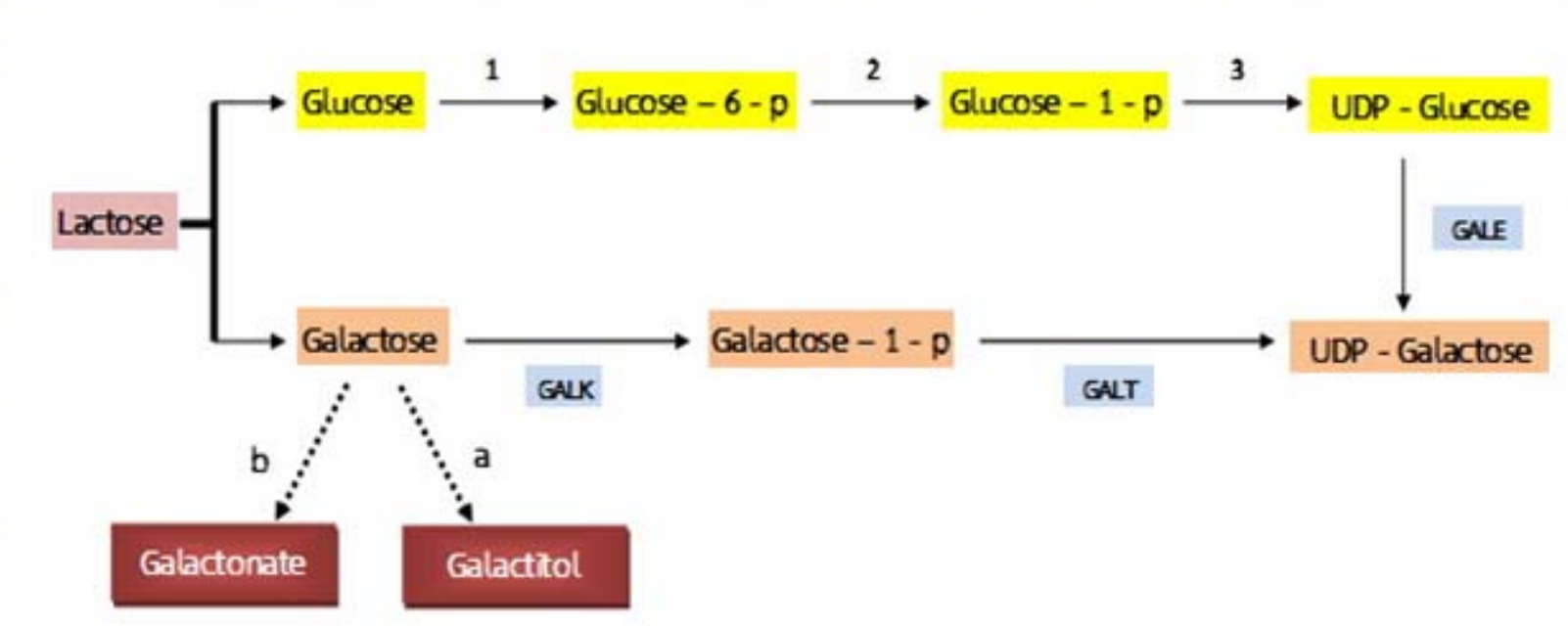

Figure 4. The Leloir pathway and alternative pathways for galactose metabolism: dotted lines a $=$ aldose reductase and $\mathrm{b}$ = pentose phosphate pathway. 1 = hexokinase, 2 = phosphoglucomutase, 3 = UDP-glucose pyrophosphorylase. GALK = galactokinase, GALT = galactose-1-phosphate uridyl transferase, GALE = galactose-6-phosphate epimerase.

Classical galactosemia is caused by a deficiency of GALT. The onset of the classical galactosemia is early and usually occurs within the first week after ingestion of galactose from milk [2-4]. The initial symptoms are nonspecific and consist of vomiting, diarrhea, lethargy, hypotonia, and poor feeding with resulting poor-weight gain. Hepatocellular injury leads to jaundice, hepatocellular insufficiency, and hepatosplenomegaly. Patients with GALT deficiency might be susceptible to Escherichia coli sepsis. Lethargy and hypotonia indicate brain toxicity. Toxic galactitol accumulation has been demonstrated in the brain tissue of neonates with classical galactosemia [6]. Cataracts are an infrequent feature of GALT deficiency. Merely $14 \%$ of patients were affected in one series, and just $20 \%$ present in the neonatal period [2]. Conversely, cataracts typically appear within GALK deficiency and GALE deficiency. In one review of the literature, cataract appeared in $75 \%$ of patients with GALK deficiency, while $5 \%$ of patients had pseudotumor cerebri or hepatosplenomegaly [5]. Clinical features of GALE deficiency vary from isolated hypergalactosemia to severe damage of selected organs resembling severe classic galactosemia and cataract, and typically appear within the first few months of life [1, 2]. By contrast with the second Thai case, our baby had no evidence of cataract [11]. Liver dysfunction is a predominant clinical manifestation. Jaundice appeared shortly after breast-feeding. In the early period, clinical features were complicated by other additional conditions, including sepsis, TPN, and G6PD deficiency and there was a limitation of hospital facilities; therefore, the diagnosis was delayed. Initial liver biopsy has been lately performed. Morphologic features indicated severe liver damage, but they were not specific and lacked of usual morphologic changes, especially microvesicular and macrovesicular steatosis, which have been described in galactosemia $[13,14]$. Therefore, many potential causes of neonatal hepatitis, including viral infection and metabolic disorders were excluded by investigations.

The appropriate treatment for an infant with galactosemia is to (1) eliminate any lactose-containing formula and breast feeding, and begin a formula such as a soy-based formula that contains no lactose as soon as possible, (2) monitor for signs of sepsis, and (3) monitor for signs of coagulopathy [3]. Newborns who are diagnosed early and remain on a galactose restricted diet usually reach adulthood. In spite of strict dietary adherence, they gradually develop a number of long-term complications: delayed language acquisition, speech defects, cognitive impairment, and learning problems, cataracts, and hypergonadotrophic hypogonadism. Most affected patients have irreversible changes liked cataract, cirrhosis, and mental retardation $[11,15]$. Growth is generally delayed in most patients, but final height-for-age is not significantly less than 
for the general population [16]. Cataracts are one of the few complications, that are produced by reduction of free galactose by aldose reduction leading to swelling of lens fibers, usually resolve with galactose restriction in early cases [17]. There is no difference in growth between infants fed a soy-based formula or cow's milk formula [18]. After galactose restriction and soy milk replacement, hepatocellular injury is less and liver function test results became normal. The present patient has slow progression of growth and development, particularly speech and language.

Galactosemia is a life-threatening disorder with emerging clinical manifestation, resulting in toxic metabolites to particular tissues or organs that occur in newborns after galactose ingestion, especially from breast milk. Immediate treatment with a galactosefree diet prevents an acute neonatal toxic state and reduces the severity of tissue injury. This disorder should be diagnosed clearly and urgently, which should be of great benefit to patients and their families in terms of patient care and quality of life. In Thailand and other Asian countries, the neonatal screening program is still unavailable; diagnosis of galactosemia might be delayed. Our case study is an example for all physicians to be aware of, so that they realize that this serious condition needs an immediate response to prevent further deterioration.

\section{Acknowledgments}

The authors would like to thank the nurse team for nursing care at Naresuan university hospital, Nithiwat Vatanavicharn, MD, for the metabolic laboratory work, and Linda Intralak for the grammatical reviewing with the manuscript. The authors have no conflict of interest to report.

\section{References}

1. Timson DJ. The structural and molecular biology of type III galactosemia. IUBMB Life. 2006; 58:83-9.

2. Broomfield AA, Brain C, Grunewald S. Galactosaemia an update. J Paediatr Child Health. 2011; 21:65-70.

3. Berry GT. Galactosemia: when is it a newborn screening emergency? Mol Genet Metab. 2012; 106: 7-11.

4. Bosch AM. Classical galactosaemia revisited. J Inherit Metab Dis. 2006; 29:516-25.

5. Bosch AM, Bakker HD, van Gennip AH, van
Kempen JV, Wanders RJ, Wijburg FA. Clinical features of galactokinase deficiency: a review of the literature. J Inherit Metab Dis. 2002; 25:629-34.

6. Leslie ND. Insights into the pathogenesis of galactosemia. Annu Rev Nutr. 2003; 23:59-80.

7. Cheung KL, Tang NL, Hsiao KJ, Law LK, Wong W, Ng PC, et al. Classical galactosaemia in Chinese: a case report and review of disease incidence. J Paediatr Child Health. 1999; 35:399-400.

8. Wasant P. Organising services for IMD in Thailand: twenty years experience. Ann Acad Med Singapore. 2008; 37:117-3.

9. Wasant P, Matsumoto I, Liammongkolkul S. Detection of inborn errors of metabolism in Thai infants via gas chromatography and mass spectrometry. Southeast Asian J Trop Med Pub Health. 1999; 30:160-5.

10. Wasant P, Svasti J, Srisomsap C, Liammongkolkul S. Inherited metabolic disorders in Thailand. J Med Assoc Thai. 2002; 85:S700-9.

11. Boonyawat B, Kamolsilp M, Phavichitr N. Galactosemia in Thai patient at Phramongkutklao Hospital: a case report. J Med Assoc Thai. 2005; 88: S275-80.

12. Siripunthana S, Sahakitrungruang T, Washarasindhu S, Suphapeetiporn K, Supornsilchai V. A discordant of blood glucose analysed by Glucometer and the Central lab method in an infant with Galactosemia. J Pediatr Endocrin. 2013; 2013:1.

13. Medline A, Medline NM. Galactosemia: early structural changes in the liver. Can Med Assoc J. 1972; 107:877-8.

14. Gareth P. Jevon JED. Metabolic Disorders in Childhood. In: Pierre Russo EDR, David A. Piccoli, editor. Pathology of Pediatric Gastrointestinal and Liver Disease: Springer Science \& Business Media; 2004. p. 270-99.

15. Ramesh S, Krishnan BR. Galactosemia. Indian Pediatr. 1991; 28:789-91.

16. Waggoner DD, Buist NR, Donnell GN. Long-term prognosis in galactosaemia: results of a survey of 350 cases. J Inherit Metab Dis. 1990; 13:802-18.

17. Ridel KR, Leslie ND, Gilbert DL. An updated review of the long-term neurological effects of galactosemia. Pediatr Neurol. 2005; 33:153-61.

18. Vandenplas Y, De Greef E, Devreker T, Hauser B. Soy infant formula: is it that bad? Acta Paediatr. 2011; 100: 162-6. 